\section{Versuchslösungen}

Sie unterscheiden sich von den Versuchslösungen früherer Arbeiten nur dadurch, daß sie immer 2.10-2-m. Cystein - ebenso wie die Extraktionslösungen - als SHSchutz enthalten. Die Leistung der Modelle wird hierdurch deutlich verbessert.

6. ATP-Spaltung wird nach $\mathrm{F}$ is $\mathrm{ke}$ und $\mathrm{Sub}$ a r row bestimmt, nachdem der frische Muskel $1 \mathrm{~min}$ und das
Wasser-Glycerin-extrahierte Material nach dem Auswaschen des Glycerins nochmals $4 \mathrm{~min}$ mit dem Blendor behandelt worden ist. Die Suspension war dann so fein, daß bis herab zu einer ATP-Konzentration von 1,5·10-4- $m$. keine Diffusionsschwierigkeiten mehr vorhanden sind.

Für Anregung zu dieser Arbeit und häufige Beratungen sind wir Herrn Prof. H. H. W e be r zu großem Dank verpflichtet.

\title{
Über den Einfluß der Wellenlänge auf die Chinonreduktion in grünen Grana
}

\author{
Von Otto Warburg \\ Aus dem Kaiser-Wilhelm-Institut für Zellphysiologie, Berlin-Dahlem \\ (Z. Naturforschg. 7 b, 443-446 [1952]; eingegangen am 25. Juli 1952)
}

\begin{abstract}
Während bei der Photosynthese dēr Quantenbedarf unabhängig von der Wellenlänge ist, ändert sich bei der Chinonreduktion der Quantenbedarf mit der Wellenlänge. — Die Energieausbeute bei der Chinonreduktion beträgt nur etwa 1\% der absorbierten Lichtenergie. Wahrscheinlich ist der energetische Mechanismus der beiden Vorgänge verschieden.
\end{abstract}

1944 wurde gefunden ${ }^{1}$, daß isolierte grüne . Grana bei Belichtung Chinon unter Sauerstoffentwicklung reduzieren:

2 Chinon $+2 \mathrm{H}_{2} \mathrm{O}=2$ Hydrochinon $+1 \mathrm{O}_{2}-52000 \mathrm{cal}$

Wir haben den Quantenbedarf dieser Reaktion bestimmt, die Anzahl Lichtquanten, die von den Grana absorbiert werden müssen, damit 1 Molekül Sauerstoff entwickelt wird. Die Wellenlängen waren 366 , 436, 480 und $644 \mathrm{~m} \mu$.

Unsere Ergebnisse gelten nur für Chinon als Wasserstoffacceptor, nicht aber für die Eisen-Reagenzien von Robert Hill2, Ferrioxalat + Ferrizyanid, die bei Zusatz zu unsern Grana keinen Sauerstoff entwickelten.

\section{Versuchsanordnung}

Grüne Grana aus Spinatblättern, nach unsrer früheren Vorschrift ${ }^{1}$ gewonnen, wurden auf der Zentrifuge bei $30000 \mathrm{~g}$ mit aus Quarz destilliertem Wasser mehrmals gewaschen und dann bei $-15^{\circ}$ in 0,05-proz. $\mathrm{KCl}$ aufbewahrt, wobei ihre photochemische Aktivität längere Zeit unverändert erhalten blieb.

Als $\mathrm{Ma} ß$ der Granamenge diente ihr Chlorophyllgehalt. $7 \mathrm{~cm}^{3}$ Grana-Suspension, die 5,6 mg Chlorophyll enthielten, wurden in Manometergefäße der Kästchenform gegeben, deren Volumen etwa $17 \mathrm{~cm}^{3}$

1 Ot to Warburg u. W. Lüttgens, Naturwiss. 32, 161 [1944]; sowie O t t o W a r b u r g, Schwermetalle, Cantor-Verlag, Freiburg 1946 u. 1948.

2 R obert Hill, Proc. Roy. Soc. [London], Ser. B 127, 192 [1939]; 129, 238 [1940]. betrug und deren Gasraum mit Argon gefüllt wurde. $4 \mathrm{mg}$ frisch sublimiertes Chinon wurde hinzugefügt. Seitenräume der Kästchen waren nicht notwendig, weder für Kalilauge, da Chinon mit gewaschenen Grana keine Kohlensäure entwickelt, noch zum Einkippen des Chinons, da die photochemische Aktivität der Grana nach Zugabe des Chinons stundenlang unverändert blieb.

Keine Druckänderungen wurden im Dunkeln beobachtet. Zur Belichtung wurde der horizontal in den Thermostaten eintretende Lichtstrahl durch einen $45^{\circ}$-Silberspiegel senkrecht nach oben in die GranaSuspension reflektiert. Die Versuchszeiten wurden so gewählt, daß die Druckänderungen etwa $+10 \mathrm{~mm}$ betrugen, die einer Sauerstoffentwicklung von $9 \mathrm{~mm}^{3}$ gleich waren. Da $4 \mathrm{mg}$ Chinon nach Gl. (1) $415 \mathrm{~mm}^{3}$ Sauerstoff entwickeln können, reichte eine Füllung für viele aufeinanderfolgende Messungen aus.

Bei der hohen Chlorophyllkonzentration von $0,8 \mathrm{mg} / \mathrm{cm}^{3}$, die einer Flächendichte von $0,73 \mathrm{mg} / \mathrm{cm}^{3}$ entsprachen, war die Lichtabsorption in jedem der 4 Spektralbezirke vollständig.

\section{W elle nlänge n}

Als Lichtquelle diente eine 500-Watt-Quecksilberhöchstdrucklampe mit Cadmiumzusatz der Osram-Studiengesellschaft Berlin. Die Quecksilberlinien 366 und $436 \mathrm{~m} \mu$ sowie die Cadmiumlinien 480 und $644 \mathrm{~m} \mu$ wurden mit Filter- und Interferenzscheiben des Glaswerks Schott, Landshut, folgendermaßen isoliert:

$366 \mathrm{~m} \mu$. $5 \mathrm{~cm}$ Wasser, $2 \mathrm{~cm} \mathrm{10-proz.} \mathrm{CuSO}_{4} \cdot 5 \mathrm{H}_{2} \mathrm{O}$, $1 \mathrm{~mm} \mathrm{UG} 1$ 
$436 \mathrm{~m} \mu .5 \mathrm{~cm}$ Wasser, $2 \mathrm{~cm} \mathrm{10-proz.} \mathrm{CuSO}_{4} \cdot 5 \mathrm{H}_{2} \mathrm{O}$, Interferenzscheibe sowie $2 \mathrm{~mm}$ BG 12

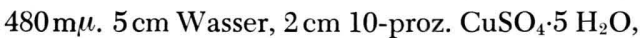
$1 \mathrm{~cm}$ Guineagrün $50 \mathrm{mg}: 100 \mathrm{~cm}^{3}$ sowie je 2 mm GG 13 und BG 12

$644 \mathrm{~m} \mu .5 \mathrm{~cm}$ Wasser, kein $\mathrm{CuSO}_{4}$,

Interferenzscheibe, $2 \mathrm{~mm}$ RG 2

Die Gesamtintensität des Lichtstrahls wurde vor dem Thermostaten bolometrisch gemessen. Der Lichtverlust auf dem Weg von der äußeren Thermostatenwand bis zum Eintritt in die Granasuspension — die „Durchlässigkeit“ D des Thermostatenwegs wurde für jeden Spektralbezirk bestimmt. D rührt her von 2 Glasscheiben mit dazwischenliegender Luftschicht, von dem mit Glas belegten Silberspiegel, von 5 Übergängen Wasser-Glas und von der Streuung an den Staubteilchen des Wasserweges und betrug z. B. für $366 \mathrm{~m} \mu$ 0,44; für $436 \mathrm{~m} \mu \quad 0,58$; für $480 \mu$ 0,62 und für $644 \mathrm{~m} \mu 0,59$.

Für jeden Spektralbezirk wurde das Intensitätsgebiet ermittelt, in dem die Ausbeute maximal und unabhängig von der Intensität war. Unter unsern Versuchsbedingungen und in unsern 4 Spektralbezirken war die Ausbeute maximal, wenn die eingestrahlte Gesamtintensität weniger als $80 \mathrm{~mm}^{3}$ Quanten pro Minute betrug. Die Flächenintensität des Lichts an der Eintrittsstelle in die Granasuspension spielte dabei, in Übereinstimmung mit früheren $\mathrm{Er}$ fahrungen, keine wesentliche Rolle.

\section{Das Verhältnis Grana/Chinon}

Gibt man zu $4 \mathrm{mg}$ Chinon, die in $7 \mathrm{~cm}^{3}$ 0,05-proz. $\mathrm{KCl}$ gelöst sind, so viel grüne Grana, daß die Lichtabsorption vollständig ist, mißt die Ausbeute und läßt dann die Granakonzentration steigen, so steigt die Ausbeute, bis etwa $6 \mathrm{mg}$ Grana-Chlorophyll zugegeben worden sind. Bei weiterem Granazusatz bleibt die Ausbeute dann konstant. Das Verhältnis Grana/Chinon ist also für die Bestimmung der maximalen Ausbeute von großer Bedeutung. Zum mindesten bei jedem Wechsel des Granamaterials muß von neuem geprüft werden, ob das Verhältnis Grana /Chinon optimal ist.

4. Bemerkungen über die ChinonReduktion

a) Wir haben uns nochmals ${ }^{1}$ davon überzeugt, daß Grana, die mit dest. Wasser mehrmals gewaschen sind - wozu man eine hochtourige Zentrifuge braucht - bei Belichtung nicht mehr imstande sind, bei Zugabe von Chinon Sauerstoff zu entwickeln; $\mathrm{da} ß$ aber kleine Mengen von Cl- oder Br-Ionen die photochemische Wirksamkeit vollständig wiederher- stellen. Auch Jod- und $\mathrm{NO}_{3}$-Ionen sind wirksam, aber weniger als Chlor- oder Brom-Ionen. Andere wirksame Anionen haben wir bisher nicht gefunden, alle Kationen, die wir prüften, waren unwirksam. Eine Erklärung dieser sehr interessanten selektiven Cofermentwirkungen ist bisher nicht gefunden worden. Im Zellsaft grüner Blätter ist das Coferment im allgemeinen Chlorion.

b) Chinon absorbiert im Blau und nimmt bei Ausbeutebestimmungen im Blau dem Chlorophyll einen Teil des Lichts fort. Aber dieser Teil ist sehr klein. Denn der Absorptionskoeffizient des Chinons für die Wellenlänge $436 \mathrm{~m} \mu$ ist von der Größenordnung $0,4 \mathrm{~cm}^{2} / \mathrm{mg}$, während er für Chlorophyll von der Größenordnung $190 \mathrm{~cm}^{2} / \mathrm{mg}$ ist. $4 \mathrm{mg}$ Chinon können also $6 \mathrm{mg}$ Chlorophyll nur $0,15 \%$ der Wellenlänge $436 \mathrm{~m} \mu$ fortnehmen.

c) Gibt man in einem Reagensglas $2 \mathrm{mg}$ Chinon und $2 \mathrm{mg}$ Hydrochinon, gelöst in je $0,2 \mathrm{~cm}^{3}$ Wasser, zusammen, so erscheint die tiefrote Farbe des Chinhydrons, die jedoch fast vollständig wieder verschwindet, wenn man mit Wasser auf $7 \mathrm{~cm}^{3}$ auffüllt (Beobachtung von oben, so daß die Substanzmenge pro $\mathrm{cm}^{2}$ konstant bleibt). Unter unsern Versuchsbedingungen also ist das Chinhydron fast vollständig dissoziiert und kann weder optisch noch sonstwie Komplikationen verursachen. Tatsächlich hat Zusatz von Hydrochinon keinen Einfluß auf die photochemische Ausbeute, weder bei Lichtsättigung, wie schon früher festgestellt ${ }^{1}$, noch bei niedrigen Lichtintensitäten, wovon wir uns im Lauf dieser Arbeit. überzeugt haben (vgl. Protokoll 3).

\section{Ergebnisse}

Je nach der Wellenlänge fanden wir einen Quantenbedarf von 60-100 Molen pro Mol entwickelten Sauerstoffs, wobei der Fehler innerhalb einer Versuchsreihe $5 \%$ nicht überstieg. Die Ausbeuten in verschiedenen Granasuspensionen waren bis zu 10\% verschieden; der Einfluß der Wellenlänge auf die Ausbeute mußte deshalb für dieselbe Granasuspension ermittelt werden. Zum Beispiel fanden wir (Protokolle 1 und 2) für eine Granasuspension an zwei aufeinanderfolgenden Tagen, wenn die Suspension bei $-15^{\circ}$ aufbewahrt wurde:

$\begin{array}{cc}\text { Wellenlänge } & \text { Quantenbedarf } \\ {[\mathrm{m} \iota]} & {\left[\frac{h v}{\mathrm{O}_{2}}\right]} \\ 366 & 65 \\ 436 & 73 \\ 480 & 85 \\ 644 & 101\end{array}$


1. Beispiel. Vergleich von 644 und $480 \mathrm{~m} \mu$.

$$
\begin{array}{llll}
\lambda 644 & J=30,2 & h \text { in } 30^{\prime}+10 \mathrm{~mm}=9 \mathrm{~mm}^{3} . & 1 / \varphi=\frac{30 \times 30,2}{9}=100 \\
\lambda 480 & J=25,6 & h \text { in } 30^{\prime}+10 \mathrm{~mm}=9 \mathrm{~mm}^{3} . & { }^{1} / \varphi=\frac{25,6 \times 30}{9}=85,4 \\
\lambda 644 & J=30,2 & h \text { in } 30^{\prime}+9,5 \mathrm{~mm}=8,6 \mathrm{~mm}^{3} . & 1 / \varphi=\frac{30 \times 30,2}{8,6}=105
\end{array}
$$

2. Beispiel. Vergleich von 644,436 und $366 \mathrm{~m} \mu$.

$$
\begin{aligned}
& \left.\begin{array}{lll}
\lambda .644 & J=30 & h \text { in } 30^{\prime}+10 \mathrm{~mm}=9 \mathrm{~mm}^{3} \\
\lambda .644 & J=30 & h \text { in } 30^{\prime}+10 \mathrm{~mm}=9 \mathrm{~mm}^{3}
\end{array}\right\}^{1 / \varphi}=\frac{60 \times 30}{18}=100 \\
& \left.\begin{array}{lll}
\lambda 436 & J=20,8 & h \text { in } 30^{\prime}+9,5 \mathrm{~mm}=8,55 \mathrm{~mm}^{3} \\
\lambda 436 & J=20,8 & h \text { in } 30^{\prime}+9,5 \mathrm{~mm}=8,55 \mathrm{~mm}^{3}
\end{array}\right\} 1 / \varphi=\frac{60 \times 20,8}{17,1}=73 \\
& \left.\begin{array}{lll}
\lambda .644 & J=30 & h \text { in } 30^{\prime}+10 \mathrm{~mm}=9 \mathrm{~mm}^{3} \\
\lambda .644 & J=30 & h \text { in } 60^{\prime}+20 \mathrm{~mm}=18 \mathrm{~mm}^{3}
\end{array}\right\} 1 / \varphi=\frac{90 \times 30}{27}=100 \\
& \lambda 366 \quad J=14,7 \quad h \text { in } 60^{\prime}+15 \mathrm{~mm}=13,5 \mathrm{~mm}^{3} \quad 1 / \varphi=\frac{60 \times 14,7}{13,5}=65 \\
& \lambda 644 \quad J=30 \quad h \text { in } 30^{\prime}+10 \mathrm{~mm}=9 \mathrm{~mm}^{3} \quad \mathrm{r} / \varphi=\frac{30 \times 30}{9}=100
\end{aligned}
$$

3. Beispiel. Vergleich verschiedener Intensitäten $\lambda .436$. Zeitliche Konstanz!

$\left.\begin{array}{llll}\lambda .436 & J=134 & h \text { in } 15^{\prime}+20,5 \mathrm{~mm}=18,5 \mathrm{~mm}^{3} & 1 / \varphi=\frac{15 \times 134}{18,5}=109 \\ & & & \\ \lambda 4436 & J=71,3 & h \text { in } 15^{\prime}+16 \mathrm{~mm}=14,4 \mathrm{~mm}^{3} & 1 / \varphi=\frac{15 \times 71,3}{14,4}=74 \\ & & & \\ \lambda 4436 & J=25,8 & h \text { in } 15^{\prime}+5,5 \mathrm{~mm}=4,95 \mathrm{~mm}^{3} & 1 / \varphi=\frac{15 \times 25,8}{4,95}=78 \\ & & & \\ \lambda .436 & J=12,6 & h \text { in } 30^{\prime}+5,5 \mathrm{~mm}=4,95 \mathrm{~mm}^{3} & 1 \\ & & \\ \lambda .436 & J=71,3 & h \text { in } 15^{\prime}+16,0 \mathrm{~mm}=14,4 \mathrm{~mm}^{3} \\ \lambda .436 & J=71,3 & h \text { in } 15^{\prime}+16,5 \mathrm{~mm}=14,9 \mathrm{~mm}^{3} \\ \lambda .436 & J=71,3 & h \text { in } 15^{\prime}+16,0 \mathrm{~mm}=14,4 \mathrm{~mm}^{3} \\ \lambda .436 & J=71,3 & h \text { in } 15^{\prime}+16,0 \mathrm{~mm}=14,4 \mathrm{~mm}^{3} \\ \lambda .436 & J=71,3 & h \text { in } 15^{\prime}+16,0 \mathrm{~mm}=14,4 \mathrm{~mm}^{3} \\ \lambda .436 & J=71,3 & h \text { in } 15^{\prime}+16,0 \mathrm{~mm}=14,4 \mathrm{~mm}^{3} \\ \lambda .436 & J=71,3 & h \text { in } 15^{\prime}+16,0 \mathrm{~mm}=14,4 \mathrm{~mm}^{3} \\ \lambda .436 & J=71,3 & h \text { in } 15^{\prime}+15,0 \mathrm{~mm}=13,5 \mathrm{~mm}^{3} \\ \lambda .436 & J=71,3 & h \text { in } 15^{\prime}+16,0 \mathrm{~mm}=14,4 \mathrm{~mm}^{3}\end{array}\right\} \quad$

Multiplizieren wir den Quantenbedarf mit dem Energieinhalt von $1 \mathrm{Mol}$ Quanten, so erhalten wir

\begin{tabular}{cc}
$\begin{array}{c}\text { Wellenlänge } \\
{[\mathrm{m} \mu]}\end{array}$ & $\begin{array}{c}\text { Quantenbedarf } \times N_{\mathrm{L}} h v \\
\text { [cal.] }\end{array}$ \\
366 & $65 \times 77500=5,04 \times 10^{6}$ \\
436 & $73 \times 65000=4,75 \times 10^{6}$ \\
480 & $85 \times 59000=5,02 \times 10^{6}$ \\
644 & $101 \times 45000=4,55 \times 10^{6}$ \\
\cline { 2 - 2 } & Mittel $4,85 \times 10^{6}$
\end{tabular}

d. h., in den 4 untersuchten Spektralbezirken wurden etwa $5 \times 10^{6} \mathrm{cal}$. zur Entwicklung von 1 Mol Sauerstoff verbraucht und es betrug die Energieausbeute

$$
\frac{52000}{5 \times 10^{6}} \times 100=1,04 \%
$$

der absorbierten Lichtenergie.
Von der Photosynthese unterscheidet sich also die Chinonreduktion in den Grana

1. durch den niedrigen Wert der Energieausbeute, die für die Photosynthese im Rot 93\%, für die Chinonreduktion in allen Spektralbezirken aber nur $1 \%$ ist;

2. durch die Änderung des Quantenbedarfs mit der Wellenlänge. Je kurzwelliger das Licht ist, d. h. je energiereicher die Quanten sind, um so weniger Quanten werden bei der Chinonreduktion verbraucht; während der Quantenbedarf bei der Photosynthese unabhängig von der Wellenlänge ist.

Beide Unterschiede sind so schwerwiegend, daß die energetischen Mechanismen der beiden Vorgänge verschieden sein müssen. 
Schließlich sei darauf aufmerksam gemacht, daß die Energieausbeute bei $480 \mathrm{~m} \mu$ fast ebenso groß ist wie bei $644 \mathrm{~m} \mu$, obwohl bei $480 \mathrm{~m} \mu$ die Hälfte des Lichts von den gelben Pigmenten absorbiert wird, während bei $644 \mathrm{~m} \mu$ die gelben Pigmente nicht absorbieren. Die Trennung der gelben Pigmente von dem Chlorophyll nach Willstaetter und den Vergleich der Lichtabsorption des Gemischs und der gelben Pigmente haben wir für den Fall unsrer Grana nach dem für Chlorella ausgearbeiteten Verfahren ${ }^{3}$ durchgeführt und dieselben Werte erhalten. Bei der Chinonreduktion ist also das von den gelben Pigmenten absorbierte Licht ebenso wirksam wie das von dem Chlorophyll absorbierte Licht.

\author{
6. Protokolle \\ (zu den Versuchen Seite 445)
}

B olometrie : $J$ ist die Quantenintensität, die in die Granasuspension eingestrahlt ist, und zwar in $\mathrm{mm}^{3}$ Quanten pro Minute $\left(1\right.$ Mikromol Quanten $=22,4 \mathrm{~mm}^{3}$ Quanten). Lichtabsorption vollständig.

M a n o metrie : $20^{\circ}$. Gasraum Argon. $v=16,56 \mathrm{~cm}^{3}$, $v_{\mathrm{F}}=7,2 \mathrm{~cm}^{3}, k_{02}=0,90 \mathrm{~mm}^{2} . h$ sind die Druckänderungen in mm Brodie.

Im $\mathrm{G}$ e f ä $\beta: 4 \mathrm{mg}$ frisch sublimiertes Chinon, Spinatgrana $5,6 \mathrm{mg}$ Chlorophyll enthaltend, $7,2 \mathrm{~cm}^{3} 0,05 \% \mathrm{KCl}$ in aus Quarz destill. Wasser.

3 O. Warburg u. E. Negelein, Z. physik. Chem. 106, 191 [1923].

\title{
Hydrazinderivate und ihre Wirksamkeit gegenüber Mycobacterium tuberculosis
}

\author{
Von Hans A. Offe, W. Siefken und G. Domagk
}

Aus dem Wissenschaftlichen Hauptlaboratorium, Leverkusen, und der Abteilung für experimentelle Pathologie und Bakteriologie, Wuppertal-Elberfeld, der Farbenfabriken Bayer

(Z. Naturforschg. 7 b, 446-462 [1952]; eingegangen am 7. Juli 1952)

Hydrazide aliphatischer und isocyclischer Carbonsäuren sowie ihre Kondensationsverbindungen mit Oxoverbindungen zeigen in vitro tuberkulostatische Wirksamkeit. Auf Grund systematischer Untersuchungen wird gezeigt, daß Hydrazinderivate der allgemeinen Formel

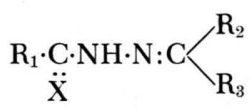

das Wachstum von Mycobacterium tuberculosis besonders gut hemmen. Für die Hemmwirkung ist der Molekülteil $\mathrm{R}_{1} \cdot \underset{\ddot{X}}{\mathrm{C}} \cdot \mathrm{NH} \cdot \mathrm{N}=$ von besonderer Bedeutung, während $=\mathrm{C}_{\mathbf{R}_{3}} \sum_{\mathrm{R}_{2}}^{\mathrm{C}}$ modifizierenden Einfluß ausübt.

1943 berichtete D om a g k ${ }^{1 \mathrm{a}}$, daß das Sulfothiodiazol ein Sulfonamid von geringer, aber deutlicher tuberkulostatischer Wirksamkeit (t. W.) ist. Der Umstand, daß B eh n is c h 1943 ein Vorprodukt zur Herstellung von Thiodiazol-Derivaten, das Benzaldehyd-thiosemicarbazon auf Grund seiner Vorstellungen von der Wirksamkeit schwefelhaltiger Hydrazinderivate zur Prüfung gab, führte dann durch die Untersuchungen von $\mathrm{B}$ e h $\mathrm{n}$ is $\mathrm{ch}, \mathrm{M}$ i et $\mathrm{zsch}$ und H. S chmidt zu einer ganzen Klasse von Verbin[1943]. dungen, den Thiosemicarbazonen (IV) ${ }^{5}$ mit hoher t.W. Dadurch wurden erstmalig wertvolle Tuberkuloseheilmittel der Öffentlichkeit auf Basis. von Hydrazinderivaten aus unseren Laboratorien zur Verfügung gestellt. - In . den Thiodiazolderivaten und den Thiosemicarbazonen liegen Hydrazinderivate,

5 R. B ehnis ch, F. Mi et z s ch, H. S chmidt u. G. D o m a g k, Naturwiss. 33, 315 [1946]; R. B e h$\mathrm{n}$ is ch, F. Mietz s h u. H. S chmidt, Angew. Chem. 60, 113 [1948]. - Vgl. ferner Deutsche Patentanmeldungen der I.G. Farbenindustrie A.-G. J 76179 , $76180,76218,76219,76679,76680,76745,77783,77784$, $78133,78134,78163,78658$ IV d/12 o (Okt. 1943 - Dez. 1944) und zahlreiche weitere. 